\title{
LITHIUM PLASMA SOURCES FOR ACCELERATION AND FOCUSING OF ULTRA-RELATIVISTIC ELECTRON BEAMS ${ }^{\mathrm{i}}$
}

\author{
P. Muggli, ${ }^{*},$, ii J.R. Hoffman, ${ }^{\dagger}$ K.A. Marsh, ${ }^{*}$ S. Wang, ${ }^{*}$ C.E. Clayton, ${ }^{*}$ T.C. Katsouleas, ${ }^{\dagger}$ \\ and C. Joshi \\ *Department of Electrical Engineering \\ University of California, Los Angeles, CA 90095, USA \\ ${ }^{\dagger}$ Department of Electrical Engineering-Electrophysics \\ University of Southern California, Los Angeles, CA 90089, USA
}

\begin{abstract}
A lithium (Li) plasma source is described that can be used for plasma acceleration as well as for electron beam focusing (plasma lens). The Li vapor with a density in the $2 \times 10^{15} \mathrm{~cm}^{-3}$ range is produced in a heat-pipe oven. The $\mathrm{Li}$ is ionized to $\mathrm{Li}^{+}$through single uv photon absorption. For the plasma wake field acceleration experiment, the plasma density $n_{p e} \approx 2-4 \times 10^{14} \mathrm{~cm}^{-3}$ is made uniform over the 1-meter long plasma [1]. A shorter length of uniform plasma density can be used as an underdense plasma lens, or the plasma density can be tapered for an underdense tapered plasma lens.
\end{abstract}

\section{INTRODUCTION}

Plasmas can sustain very large amplitude electric fields and are thus very suitable for particle acceleration (longitudinal field) and/or particle focusing (transverse field). In a recently proposed experiment known as E-157 [2], the $30 \mathrm{GeV}$ electron beam of the Stanford Linear Accelerator Center (SLAC) Final Focus Test Beam (FFTB) is sent in a plasma with an electron density $n_{p e}$ in the $2-4 \times 10^{14} \mathrm{~cm}^{-3}$ range. Numerical simulations show that that the electron bunch excites a wake (longitudinal plasma wave) with a maximum amplitude of $1 \mathrm{GV} / \mathrm{m}$, making it possible for the first time to accelerate electrons by $1 \mathrm{GeV}$ over 1 meter. The electrons of the bulk of the electron beam drive the wake and loose about $200 \mathrm{MeV} / \mathrm{m}$, while the trailing electrons of the same bunch experience the acceleration and gain about $1 \mathrm{GeV} / \mathrm{m}$. In previous self-modulated laser wake field acceleration (SM-LWFA) experiments fields in excess of $100 \mathrm{GV} / \mathrm{m}$ where excited but only over a few millimeters [3]. In plasma beat wave acceleration (PBWA) experiments a field of $3 \mathrm{GV} / \mathrm{m}$ has been excited over $1 \mathrm{~cm}$ [4]. the resulting electron energy gains were $\approx 100 \mathrm{MeV}$ and $30 \mathrm{MeV}$ respectively. The E-157 experiment operates in the "blow-out" regime, and the focusing force exerted by the ion column onto the beam electrons corresponds to a focusing strength in excess of $6 \times 10^{5} \mathrm{G} / \mathrm{cm}$. A shorter section of plasma $(L \approx 25 \mathrm{~cm})$ can be used as an effective underdense thick-plasma lens. Alternatively, the ionizing laser beam can be focused into the plasma to produce a tapered plasma density suitable for an underdense plasma lenses. Plasma lenses used at the final focus may significantly improve the beam

${ }^{i}$ Work supported US DoE under Grants No. DE-FG03-92ER40727 and No. DE-FG03-92ER40745, and by the NSF under Grants No. ECS9632735 and No. ECS-9617089.

ii E-mail: muggli@ee.ucla.edu luminosity and can be used to minimize beamstrahlung. The Li plasma sources combine the ease of ionization and uniformity of the Li vapor (ionization potential $5.3 \mathrm{eV}$ ), with the flexibility of a 1-photon laser induced ionization.

\section{LI PLASMA SOURCE FOR ELECTRON ACCELERATION}

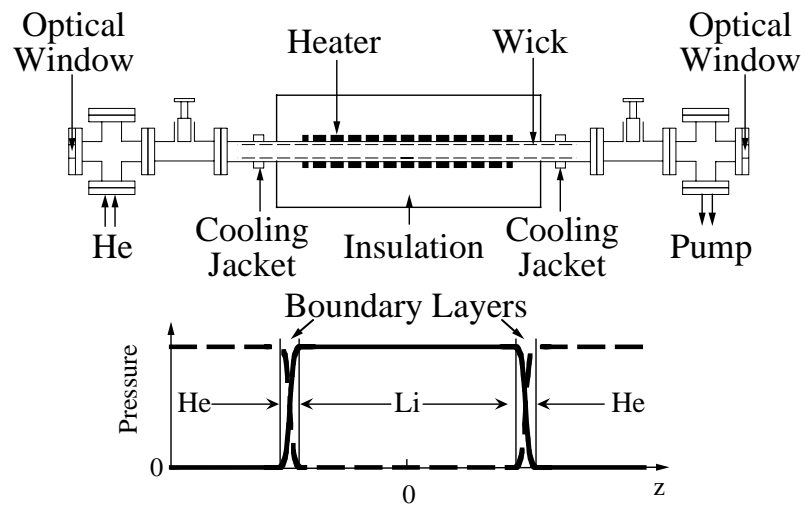

Figure 1: Schematic of the Li vapor/plasma source (not to scale).

Lithium is chosen for the plasma because it has a low ionization potential $(5.3 \mathrm{eV})$ and can thus be ionized over a large volume through a single uv photon absorption process $(\lambda<242 \mathrm{~nm})$. Li also has a relatively low atomic number $(Z=3)$ which minimizes the additional plasma density that is produced through impact ionization by the electrons of the beam to be accelerated. A neutral Li vapor density in the $10^{15}$ to $10^{16} \mathrm{~cm}^{-3}$ range can be obtained by heating the $\mathrm{Li}$ to around $750^{\circ} \mathrm{C}$. The $\mathrm{Li}$ vapor column is produced in a heat-pipe oven [5] (Fig. 1). The hot Li vapor is confined to the heated central part of the heat-pipe by the room temperature He buffer gas located at the ends of the heat-pipe. The vapor temperature is such that the $\mathrm{Li}$ vapor pressure is equal to the He pressure (in absence of significant flow). The Li density is thus determined by the adjustable He pressure. The length of the $\mathrm{Li}$ column $L$ is approximately equal to the length of the heated section of the pipe, and can be varied around that value by changing the heating power delivered to the oven. A vapor density $n_{0}=2 \times 10^{15} \mathrm{~cm}^{-3}$ was produced over $L=25 \mathrm{~cm}$ in a short oven at a temperature of $\approx 750^{\circ} \mathrm{C}[6]$. The product $n_{0} L$ was measured using the hook method [7], and by uv and by white light absorption, whereas the column length was deduced from longitudinal temperature profiles measurements.

The vapor was ionized by single photon absorption of a $20 \mathrm{~ns}, F_{0}=145 \mathrm{~mJ} / \mathrm{cm}^{2}$ (maximum) ArF uv laser pulse 
$(\lambda=193 \mathrm{~nm})$. The line integrated plasma density $n_{p e} L$ was measured by uv absorption, and by $\mathrm{CO}_{2}$ and visible laser interferometry. The plasma varied linearly with $F_{0}$ (Fig. 2) and reached a maximum of $2.96 \times 10^{14} \mathrm{~cm}^{-3}$ (limited by $F_{0}$ ) corresponding to a $15 \%$ fractional ionization. The time for the plasma density to decrease by a factor of two from its maximum value was $12 \mu \mathrm{s}$. The plasma source has reached all the required parameters for the E-157 experiment (Table 1).

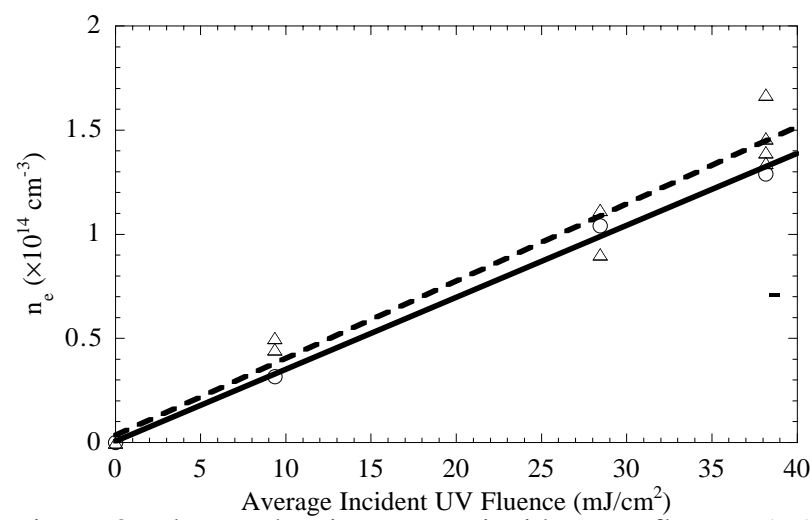

Figure 2: Plasma density versus incident uv fluence $\left(F_{0}\right)$, as measured by $\mathrm{CO}_{2}$ laser interferometry (triangles), and by uv absorption (circles). The two lines are the best linear fits to the two measurements.

Table 1: Plasma parameters required for the E-157 experiment.

\begin{tabular}{||l|l|l||}
\hline Plasma Density & $n_{p e}$ & $2-4 \times 10^{14} \mathrm{~cm}^{-3}$ \\
\hline Plasma Length & $L$ & $\approx 1 \mathrm{~m}$ \\
\hline Density Uniformity & $\Delta n_{p} / n_{p e}$ & $<25 \%$ \\
\hline Ionization Fraction & $n_{p e} / n_{0}$ & $>15 \%$ \\
\hline Radius & $r$ & $>400 \mu \mathrm{m}$ \\
\hline
\end{tabular}

For that experiment however, the length of the plasma has to be of the order of one meter for the electrons to gain $1 \mathrm{GeV}$. More importantly, the product $n_{p e} L$ has to be matched to an integer number of betatron wavelengths of the electron beam envelope in the plasma. The $\beta$-function $\beta_{b}$ of electron beam is not matched to the plasma $\beta_{p e}$,: from (Table 2) $\beta_{b}=\sigma^{2} / \varepsilon_{x}=5.6 \mathrm{~m}>\beta_{p e}\left(n_{p e}=4 \times 10^{14} \mathrm{~cm}^{-3}\right)=$ $(2 \gamma)^{1 / 2} c / \omega_{p e}=0.09 \mathrm{~m}$, where $\gamma$ is the relativistic factor of the electron beam and $\omega_{p e}=\left(n_{p e} e^{2} / \varepsilon_{0} m_{e}\right)^{1 / 2}$ is the plasma frequency. The beam envelope thus experiences betatron oscillations with a wavelength of $\lambda_{b} / 2 \approx 29 \mathrm{~cm}$, where $\lambda_{b}=2 \pi c /(2 \gamma)^{1 / 2} \omega_{p e}$ is the betatron wavelength. The beam emerges from the plasma collimated only if $L=m \lambda_{b} / 2$, $m=1,2, \ldots$ The beam size at the pinch is $\sigma_{x}^{*} \approx\left(2 \gamma c^{2} / \omega_{p e}^{2} \beta_{b}\right)^{1 / 2} \sigma_{x} \approx 1.73 \mu \mathrm{m} \quad\left(n_{p e}=4 \times 10^{14} \mathrm{~cm}^{-3}\right)$, indicating that a shorter plasma could be used as an effective plasma lens.

A 1 -meter long source $\left(\approx 3 \lambda_{b} / 2\right)$ is presently built for the E-157 experiment, in collaboration with the Laurence Berkeley Laboratory [1], while a 1.5 -meter source $\left(\approx 5 \lambda_{b} / 2\right)$ is being developed at UCLA for further experiments. In the 1-meter plasma the gradient in $n_{p e}$ resulting from the uv photon absorption can be compensated for by double passing the laser pulse through the $\mathrm{Li}$ column. For example the $n_{p e}$ variation is about $30 \%$ over $L=1 \mathrm{~m}$ for $n_{0}=2 \times 10^{15} \mathrm{~cm}^{-3}$ with an average plasma density of $\approx 2.9 \times 10^{14} \mathrm{~cm}^{-3}$ in the single pass case (incident fluence $F_{0}=100 \mathrm{~mJ} / \mathrm{cm}^{2}$ ) and is reduced to $\approx 8 \%$ while the plasma density is increased by more than $50 \%\left(4.8 \times 10^{14} \mathrm{~cm}^{-3}\right)$ by double passing the oven. Alternatively the plasma density gradient can be minimized by focusing the ionizing laser beam. In a short plasma $\left(\sigma n_{0} L<<1\right)$ the uv photons absorption is linear with distance $\left(F=F_{0} \exp \left(-\sigma n_{0} L\right) \approx F_{0}(1-\right.$ $\left.\sigma n_{0} L\right)$ and can be exactly compensated for by a cylindrical focusing.

Table 2: Electron beam parameters at the interaction point.

\begin{tabular}{||l|l|l||}
\hline \hline Number of Electrons & $N_{e}$ & $3.5-4.0 \times 10^{10}$ \\
\hline Initial Energy & $E_{0}$ & $30 \mathrm{GeV}$ \\
\hline Bunch Length & $\sigma_{z}$ & $0.6 \mathrm{~mm}$ \\
\hline Bunch Size $\left(@ 1 \times 10^{10} \mathrm{e}^{-}\right)$ & $\sigma_{x}$ & $\approx 75 \mu \mathrm{m}$ \\
\hline & $\sigma_{v}$ & $\approx 75 \mu \mathrm{m}$ \\
\hline Emittance & $\gamma \varepsilon_{x}$ & $60 \mathrm{~mm}-\mathrm{mrad}$ \\
\hline & $\gamma \varepsilon_{v}$ & $15 \mathrm{~mm}-\mathrm{mrad}$ \\
\hline
\end{tabular}

\section{BEAM FOCUSING, UNDERDENSE UNIFORM PLASMA LENS}

The leading electrons of a long electron bunch $\left(\sigma_{z}>c / \omega_{p e}\right)$ expel the plasma electrons in a radius of $\approx c / \omega_{p e}$. In the case where the beam density $n_{b}$ is larger than the plasma density $\left(n_{b}>n_{p e} / 2\right.$, the underdense plasma lens case), the core of the electron beam experiences a focusing force originating from the plasma ion column $\left(n_{p i}=n_{p e}\right)$. The force can be calculated from Poison's equation and varies linearly with radius. Its strength is $W=e n_{p e} / 2 \varepsilon_{0} c \approx 3 n_{e}\left[\mathrm{~cm}^{-3}\right] / 10^{15} \mathrm{MG} / \mathrm{cm}$. For comparison, the maximum strength of a typical magnetic quadrupole lenses is $\approx 10 \mathrm{kG} / \mathrm{cm}$. The plasma acts on the electron beam as a strong, ideal lens. The lens modifies the minimum $\beta$-function of the incoming beam according to:

$$
\frac{\beta^{*}}{\beta_{0}^{*}}=\frac{1}{1+K\left(\beta_{0}-\beta_{L}\right) \beta_{0}^{*}}
$$

where $\beta_{0}{ }^{*}$ and $\beta^{*}$ are the minimum $\beta$-functions for the beam without and with the plasma lens respectively, $\beta_{0}$ is the $\beta$-function at the lens entrance, and $K=W e / \gamma m_{e} c$. The $\beta$-function of the beam at the lens exit $\beta_{L}$ is given by:

$$
\begin{aligned}
\beta_{L} & =\frac{\beta_{0}}{2}+\frac{1}{2 K \beta_{0}^{*}}+\left(\frac{\beta_{0}}{2}-\frac{1}{2 K \beta_{0}^{*}}\right) \cos (2 \sqrt{K} L) \\
& -\frac{2 s_{0}}{2 \sqrt{K} \beta_{0}^{*}} \sin (2 \sqrt{K} L)
\end{aligned}
$$

where $s_{0}=\left(\beta_{0}{ }^{*}\left(\beta_{0}-\beta_{0}{ }^{*}\right)\right)^{1 / 2}$ is the distance between the lens entrance and the beam focus in absence of the lens. The new beam focus is located a distance $s=\left(\beta^{*}\left(\beta_{L}-\beta^{*}\right)\right)^{1 / 2}$ away from the lens exit.

The Li plasma source described in the previous section could be used to produce a $10 \mathrm{~cm}$ long plasma with a uniform density of $n_{p e}=2 \times 10^{14} \mathrm{~cm}^{-3}$ corresponding to $c / \omega_{p e}=375 \mu \mathrm{m}$. The FFTB pulse stretched to $\sigma_{z}=1.0 \mathrm{~mm}$ $\left(n_{b}=4.5 \times 10^{14} \mathrm{~cm}^{-3}>n_{p e} / 2\right.$ with $N_{e}=4 \times 10^{10}$ electrons per bunch) would focus to $\sigma_{x}=2.5 \mu \mathrm{m}$ (reduction by a factor $\approx 30) 4.0 \mathrm{~cm}$ away from the lens exit.

\section{BEAM FOCUSING, UNDERDENSE TAPERED PLASMA LENS}

In the uniform plasma lens example described in the previous section, the plasma is underdense at the lens entrance and becomes more and more underdense as the 
electron beam focuses. In order to increase the strength of the plasma lens and reduce the final beam spotsize, the plasma density can be increased along the electron beam path while keeping the plasma underdense for the focusing beam.[8] With the plasma source described in Section 2 this can be achieved by sending a de-focusing ionizing laser beam in the opposite direction to that of the electron beam. In this case, the plasma density decreases along the laser beam path (i.e., increases along the electron beam path), because of the depletion of the uv photons and because of the decrease in uv fluence resulting from the beam de-focusing. The uv photons are absorbed following $N(z)=N_{0} \exp \left\{-\sigma n_{0}(L-z)\right\}$ for $0 \leq z \leq L$, where $N_{0}=E_{0} / h v, E_{0}$ is the incident uv energy, and $h v$ the energy per photon. The plasma density created by ionization is given by $n_{p e}(z)=(1 / A)(-\partial N / \partial z) \quad=\left(\sigma n_{0} / h v\right)\left(N_{0} / A(z)\right) \exp \left\{-\sigma n_{0}(L-z)\right\}$ where $A$ is the laser beam spot size. For a thin plasma $\left(\sigma n_{0} L<<1\right)$, and a beam expanding from an initial radius $r_{0}$ to a final radius in the plasma of $r_{0}+\Delta r$ according to $r(z)=r_{0}(1+\Delta r(L-z) / L)$, the plasma density is thus:

$$
n_{p e}(z)=\frac{\sigma n_{0}}{h v} \frac{E_{0}}{\pi r_{0}^{2}} \frac{\left(1-\sigma n_{0}(L-z)\right)}{(1-\Delta r(L-z) / L)^{2}}
$$

The laser fluence incident upon the $\mathrm{Li}$ vapor is $F_{0}=E_{d} / \pi_{0}{ }^{2}$ where $\pi \mathrm{r}_{0}^{2}$ is the laser spotsize when entering the plasma. The envelope equation for the beam spot size $\sigma$ :

$$
\sigma^{\prime \prime}(z)+K(s) \sigma(z)=\frac{\varepsilon^{2}}{\sigma^{3}(s)}
$$

can be solved numerically for a plasma density profile given by eq. 4 . Figure 3 shows the beam envelope evolution for the case described in the previous section ( $n_{p e}=2 \times 10^{14} \mathrm{~cm}^{-3}$ over $L=10 \mathrm{~cm}$, sharp boundaries). Figure 4 shows the beam envelope evolution in a $10 \mathrm{~cm}$ long plasma with a plasma density tapered from 2 to $10 \times 10^{14} \mathrm{~cm}^{-3}$ by defocusing the ionizing laser. The initial vapor density is $n_{0}=2 \times 10^{15} \mathrm{~cm}^{-3}$, and the initial fluence $F_{0}=286 \mathrm{~mJ} / \mathrm{cm}^{2}$. The tapered plasma lens remains underdense over its entire length, and the final spot size is reduced from $2.5 \mu \mathrm{m}$ (in the constant $n_{p e}$ case) to $1.24 \mu \mathrm{m}$. The focal point is now located $5.0 \mathrm{~cm}$ away from the plasma exit. Also shown on these figures the same calculations for a plasma density including more realistic continuous vacuum/plasma boundaries. In this case the lens focusing strength is kept approximately the same as in the sharp boundary case by keeping the number of particles in the plasma the same in the sharp and continuous boundaries cases. Note that numerical simulations are required to confirm and optimize the examples presented here.

\section{SUMMARY}

A lithium plasma source is described that will be used to demonstrate acceleration of ultra-relativistic electrons by $\approx 1 \mathrm{GeV}$ over $\approx 1 \mathrm{~m}$. A shorter version $(\approx 10 \mathrm{~cm})$ of the same plasma source could be used as an underdense plasma lens. In the examples described here the SLAC FFTB spot size is reduced from 75 to $2.5 \mu \mathrm{m}$ in the constant plasma density case, and from $75 \mu \mathrm{m}$ to $1.25 \mu \mathrm{m}$ in the case of the plasma density tapered along the electron beam path ( 2 to $10 \times 10^{14} \mathrm{~cm}^{-3}$ ). The plasma density taper is obtained by defocusing the ionizing laser pulse.

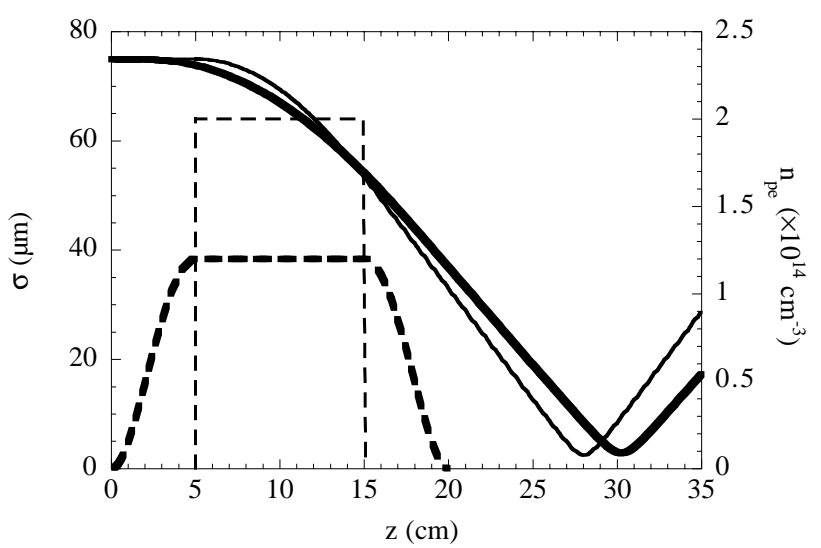

Figure 3: Evolution of the beam envelope $\sigma$ (continuous lines) when traversing an $n_{p e}=2 \times 10^{14} \mathrm{~cm}^{-3}, \quad L=10 \mathrm{~cm}$ (dashed lines) underdense plasma lens with (thick lines) and without (thin lines) continuous plasma boundaries. The total number of charges in the plasma is the same in both cases. The initial $\sigma$ is $75 \mu \mathrm{m}$, and the minimum $\sigma$ is $2.5 \mu \mathrm{m}$ in both cases, 10 respectively $13 \mathrm{~cm}$ away from the lens exit.

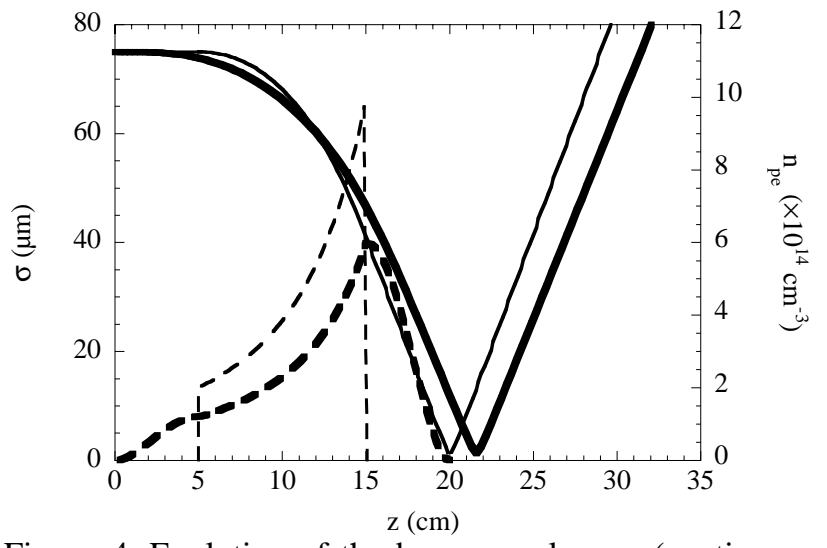

Figure 4: Evolution of the beam envelope $\sigma$ (continuous lines) when traversing an plasma with a density $n_{p e}$ tapered from 2 to $10 \times 10^{14} \mathrm{~cm}^{-3}$ over $L=10 \mathrm{~cm}$ (dashedlines) with (thick lines) and without (thin lines) continuous plasma boundaries. The plasma remains underdense along the lens. The initial $\sigma$ is $75 \mu \mathrm{m}$, and the minimum $\sigma$ is $1.24 \mu \mathrm{m} 5$ respectively $2.5 \mathrm{~cm}$ away from the lens exit.

\section{REFERENCES}

[1] S. Dimaggio et al., these proceedings.

[2] T. Katsouleas et al., Proceedings of the Particle Accelerator Conference, Vancouver, Canada, May 1997.

[3] D. Gordon et al., Phys. Rev. 80, 2133 (1998), C.I. Moore et al., Phys. Rev. Lett. 79, 3909 (1997).

[4] C.E. Clayton et al., Phys. Rev. Lett. 70, 37 (1993).

[5] C.R. Vidal et al., ,' J. Appl. Phys. 40, 3370 (1969).

[6] P. Muggli et al., to be published in IEEE Trans. Plasma Science, June (1999).

[7] W.C. Marlow, Applied Optics 6, 1715 (1967).

[8] T.C. Katsouleas, and C.H. Lai, Proc. Adv. Acc. Conc. Workshop, AIP 279, 551 (1992). 